\section{In vitro microfluidic modelling of the human blood-brain-bar- rier microvasculature and testing of nanocarrier transport}

\author{
Marco Campisi, ${ }^{1}$ Sharon W. L. Lee, ${ }^{2-4}$ \\ Tatsuya Osaki,5,6 Luca Possenti, ${ }^{7}$ \\ Clara Mattu, ${ }^{1}$ Giulia Adriani, ${ }^{4}$ \\ Valeria Chiono, ${ }^{1}$ Roger D. Kamm 5 \\ 1Department of Mechanical and \\ Aerospace Engineering, Politecnico di \\ Torino, Turin, Italy; ${ }^{2}$ Singapore-MIT \\ Alliance for Research and Technology, \\ Singapore; ${ }^{3}$ NUS, YLL School of \\ Medicine, Singapore; ${ }^{4}$ Singapore \\ Immunology Network, A*STAR, \\ Singapore; 5Department of Biological \\ and Mechanical Engineering, MIT, \\ Cambridge, MA, USA; 'Institute of \\ Industrial Science, The University of \\ Tokyo, Japan; 7Department of Materials \\ and Chemical Engineering, Politecnico \\ di Milano, Milan, Italy
}

\section{Abstract}

The blood-brain barrier (BBB) protects the brain from pathogens but also hinders drug delivery to the central nervous system. Most of the BBB models developed up to date failed to reproduce the human anatomical complexity of brain barriers, contributing to less predictive experimental platforms and poor patient outcomes. To overcome those limitations, the development of reliable in vitro models represents a crucial step towards more effective therapies. This contribution was focused on the development of an in vitro microfluidic model of the BBB able to replicate the human neurovascular organization. The microfluidic model included human induced pluripotent stem cell-derived endothelial cells, brain pericytes, and astrocytes as self-assembled microvascular networks in a 3-dimensional fibrin gel. As previously demonstrated, the BBB model exhibited perfusable and selective microvasculature, with permeability lower than conventional in vitro models and comparable with in vivo rat brain. Permeability of polystyrene nanoparticles (NPs) and synthesized polyurethane NP was measured across the BBB model as compared to conventional Transwell assays. This physiologically relevant BBB model offers an innovative and valuable platform to preclinically predict transport efficacy of drugs and carriers.

\section{Introduction}

The blood-brain-barrier (BBB) is a selective barrier that help to maintain brain homeostasis, however it also constitutes a nearly-impenetrable obstacle against therapeutic delivery to the central nervous system. Since only small compounds can cross the $\mathrm{BBB}$, this reduces the treatments available for neurodegenerative diseases and cancer. ${ }^{1}$ Polymer-based nanoparticles (NPs), due to their small size and surface functionalization potential, have emerged as a solution to deliver therapeutic cargo across the BBB. Although these techniques are considered the gold standard, current models, such as transwell or mouse models, fail to reproduce the anatomical complexity of the human BBB. Indeed, $80 \%$ of drug candidates that were successful tested in vitro and in animals later failed in clinical trials. ${ }^{2}$ Moreover, as transport across the $\mathrm{BBB}$ represents the first evidence of NP delivery capabilities, the development of a in vitro testing platform and method for quantifying NP transport behavior provides an invaluable tool to assess therapeutic efficacy.

For these reasons, a cost-effective and reliable in vitro BBB model and method that adequately reflects human in vivo conditions is required.

\section{Materials and Methods}

PDMS microfluidic devices were designed (Autocad) and fabricated by soft lithography. To develop our 3D BBB model, human induced pluripotent stem cell-derived endothelial cells (iPSC-ECs, CDI), primary astrocytes and pericytes (ScienCell) were suspended in fibrin gel (3 $\mathrm{mg} / \mathrm{mL}$ ), injected into the device, incubate to allow the gel polymerization and supported with culture medium (Vasculife) (Figure 1a). ${ }^{3}$ RT-PCR, vascular permeability and immunocytochemistry assays were performed. Polystyrene NPs (PS-NPs) (FluoSpheres) and synthesized polyurethane NPs (PU-NPs) transport was preliminary evaluated across the human BBB model. 3D permeability was quantified by the increase in fluorescence intensity in the region outside the BBB vasculature in confocal z-stack images (Figure 1b-d).

\section{Results}

This in vitro microfluidic model of the BBB containing human induced pluripotent stem cell-derived endothelial cells, brain
Correspondence: Valeria Chiono, Department of Mechanical and Aerospace Engineering, Politecnico di Torino, Turin, Italy.

E-mail: valeria.chiono@polito.it

Roger D. Kamm, Department of Biological and Mechanical Engineering, MIT, Cambridge, MA, USA.

E-mail: rdkamm@mit.edu

Key words: Human blood-brain-barrier; in vitro testing platform, microfluidic device; self-organized microvasculature; polymer nanoparticles.

Acknowledgements: The joint Ph.D. project (BIOMODE, Compagnia San Paolo), NSF, NRF, SMART BioSyM, Rocca Foundation, EU Horizon 2020 program MSCA (658665) are acknowledged.

Conference presentation: this paper was presented at the Second Centro 3R Annual Meeting - 3Rs in Italian Universities, 2019, June 20-21, University of Genoa, Italy.

Received for publication: 28 October 2019. Accepted for publication: 11 November 2019

This work is licensed under a Creative Commons Attribution NonCommercial 4.0 License (CC BY-NC 4.0).

(C) Copyright: the Author(s), 2019

Licensee PAGEPress, Italy

Biomedical Science and Engineering 2019; 3(s3):105 doi:10.4081/bse.2019.105

pericytes, and astrocytes as self-assembled microvasculature supported in fibrin gel matrix. Gene expression of tight junctions (ZO-1, occludin, and claudin-5), extra-cellular matrix proteins (Laminin and Collagen IV), and membrane transporters (PG-P, LAT1, LRP1) was higher in tri-culture condition consistently with quantitative immunocytochemistry analysis indicating BBB-like maturation. Laser confocal microscopy validated microvessel-pericytes/astrocytes contact-interactions. Characterization of microvascular network parameters as vascular diameter, branches length and vascular network area coverage were lower when including pericytes and astrocytes. This revealed that morphological changes were induced by not only the secretion of pro-angiogenic and vasculogenic growth factors but also contact signaling between cells. The BBB model exhibited perfusable and selective microvasculature created within 5-7 days (Figure 1d), showing permeability coefficient comparable to previous models and similar to in vivo measurements in rat brain. ${ }^{3}$

This established $3 \mathrm{D}$ in vitro model of the human BBB might be exploited to eval- 
a

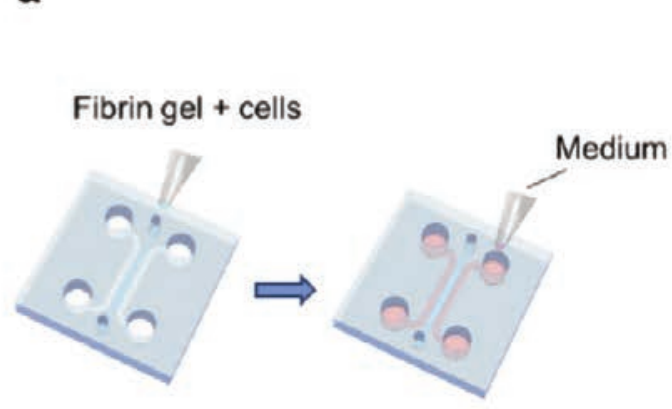

d

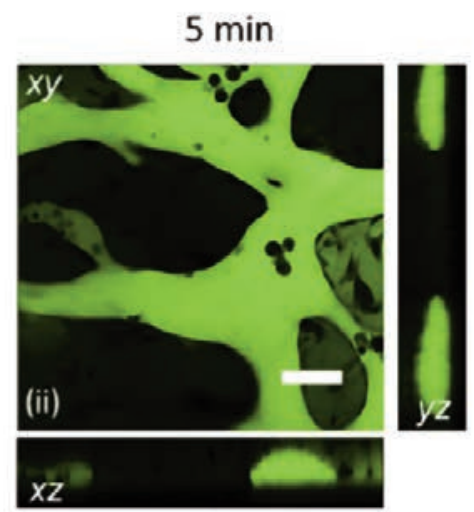

b

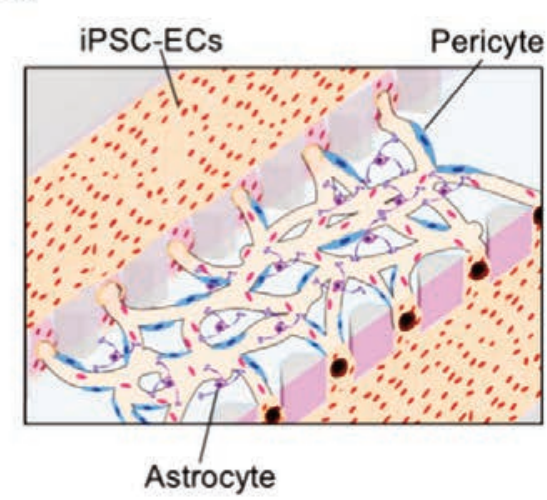

C

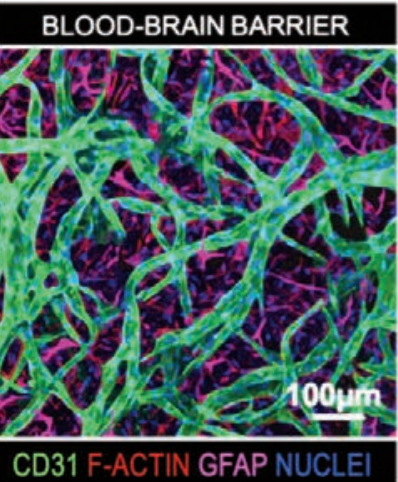

Figure 1. Representative images of the BBB model: (a) microfluidic device seeding; (b) the microvasculature obtained by triculture; (c) immunohistochemistry analysis of the BBB microvasculature; (d) confocal microscope analysis of the BBB microvasculature after perfusion with fluorescent dextran. Reproduced with permission from Campisi et al. 3

uate nanocarrier permeability such as nanoparticles. Indeed, ongoing experiments are showing that the $3 \mathrm{D}$ BBB model might be capable to elucidating differences in $3 \mathrm{D}$ NP transport between PS-NPs and PU-NPs compared to Transwell assays.

\section{Conclusions}

The BBB microfluidic model has the main advantage over other BBB-on a chip models, that all three cell types are seeded simultaneously into a single 3D gel region, producing a perfusable vascular network with permeabilities lower than those of other published models. The contribution of co-culture with pericytes and astrocytes also improved BBB formation and maturation. It also helped to regulate the upregulation of tight junction proteins and mem- brane transporters by the iPS endothelial cells, highlighted as potential targets to enhance the penetration of drugs into the brain.

The work also presents an innovative translational application of the 3D $\mathrm{BBB}$ microvascular model to assess nanocarrier transport and quantify the permeability of different nanocarriers that cross the human BBB. ${ }^{4}$ A permeability method is being optimized in order to perform pre-clinical screening of drug candidates within a physiologically-relevant BBB microvasculature, reducing the use of animal models.

This robust $3 \mathrm{D}$ BBB microvascular model could be potentially applied to patient-specific and neurodegenerative diseases modelling, offering a novel platform to study both drug transport for preclinical screening as well as neurovascular functions within a physiologically-relevant BBB microvasculature.

\section{References}

1. Pardridge WM. CSF, blood-brain barrier, and brain drug delivery. Expert Opin Drug Deliv 2016;13:963-75.

2. Crawford L, Rosch J, Putnam D. Concepts, technologies, and practices for drug delivery past the blood-brain barrier to the central nervous system. J Control Release 2016;240:251-66.

3. Campisi M, Shin Y, Osaki T, et al. 3D self-organized microvascular model of the human blood-brain barrier with endothelial cells, pericytes and astrocytes. Biomaterials 2018;180:117-29.

4. Hajal C, Campisi M, Mattu C, et al. In vitro models of molecular and nanoparticle transport across the blood-brain barrier. Biomicrofluidics 2018;12: 042213. 